\title{
Parametric resonance of acoustic and optical phonons in a GaAs/GaAsAl quantum well in the presence of a laser field
}

\author{
Nguyen Tien Dung, ${ }^{* 1}$ Le Canh Trung, ${ }^{1}$ Pham Thi Hoai Duong, ${ }^{1}$ Tran Cong Phong ${ }^{2}$ \\ ${ }^{1}$ Lab for Photonic Crystal Fiber, Vinh University, 182 Le Duan Streer, Vinh City, Viet Nam \\ ${ }^{2}$ The Vietnam Institute of Educational Sciences, No. 101, Tran Hung Dao Str., Hoan Kiem Dist., Hanoi, Viet Nam
}

Received October 25, 2020; accepted March 28, 2021; published March 31, 2021

\begin{abstract}
In this paper, we analytically investigated the possibility of parametric resonance of acoustic and optical phonons. We obtained a general dispersion equation for parametric amplification and transformation of phonons. The dispersions of resonant acoustic phonon modes and threshold amplitude of the field are obtained for acoustic phonon parametric amplification. The parametric amplification for acoustic phonons in a $\mathrm{GaAs} / \mathrm{GaAsAl}$ quantum well can occur under the condition that the amplitude of an external electromagnetic field is higher than threshold amplitude.
\end{abstract}

Resonance effects, in general, and parametric resonance are important processes in physics research. There are many resonance processes that we try to strengthen due to their beneficial effects but there are also processes that people try to limit or eliminate. The study of parametric resonance and parameter enhancement in low-dimensional systems is expected to provide a broad base for many applications in modern physics and engineering, especially in low-dimensional materials engineering, microelectronics technology, information technology $[1 \div 2]$.

The resonance of phonon parameters and optical phonons in conventional semiconductor semiconductors in the presence of electrons has been studied in recent years $[3 \div 5]$. In this paper, we study the parametric resonance between acoustic phonons in a GaAs/GaAsAl quantum well (QW) [6] in the case of degenerate electron gas, from which we find the dispersion resonance frequency and amplitude condition of a laser field for this resonance.

We use a simple model for a $\mathrm{QW}$, in which a twodimensional electron gas is confined by the infinite potential $U(z)$ of a square well along the $z$ direction and electrons are free on the $x-y$ plane. Suppose the electromagnetic wave propagates along the axis of the $\mathrm{Oz}$ axis of a square well, the vector of electric field strength of an electromagnetic wave takes the form $\stackrel{1}{E}=\stackrel{1}{E_{0}} \sin (\Omega t), \stackrel{\stackrel{1}{E}}{{ }_{0}}$ direction perpendicular to the axis Oz. The electron energy is quantized and each state of the electron is characterized by the quantization index $n$ and

*E-mail: tiendungunivinh@gmail.com the wave vector $k_{\perp}$ directed perpendicularly to the axis $\mathrm{O} z$. Hamiltonian of the electron-phonon system when a laser field is present [7]:

$$
\hat{H}(t)=\hat{H}_{0}(t)+\hat{H}_{e-a c}+\hat{H}_{e-o p},
$$

where $\hat{H}_{0}(t)$ is the Hamiltonian of the electron-phonon system that does not interact with the laser field:

$$
\hat{H}_{0}(t)=\sum_{n} \varepsilon_{n}\left(\stackrel{\mathrm{r}}{k_{\perp}}-\frac{e}{\mathrm{~h} c} \stackrel{\mathrm{r}}{A}(t)\right) a_{n}^{+} a_{n}+\sum_{q} \mathrm{~h} \omega_{q}^{\mathrm{r}} b_{q}^{+} b_{q}^{\mathrm{r}}+\sum_{q} \mathrm{~h}{ }_{q}^{\mathrm{r}} c_{q}^{+} c_{q}^{\mathrm{r}},
$$

where $|n\rangle=\left|k_{\perp}^{\mathrm{r}}, n\right\rangle$ is the electron state corresponding to the wave vector $\stackrel{k}{k}_{\perp} ; a_{n}^{+}, a_{n}$ are the creation and annihilation operators of electron in the $|n\rangle$ state; $b_{q}^{+}, b_{q}^{\text {r }}$ and $c_{q}^{+}, c_{q}^{r}$ are the creation and annihilation operators of acoustic phonon and optical phonon with wave vector $\stackrel{\mathrm{r}}{q}$; $\omega_{q}^{\mathrm{r}}, \nu_{q}^{\mathrm{r}}$ are the frequency of acoustic phonon and optical phonon; $\mathrm{c}$ is the speed of light in a vacuum; $\varepsilon_{n}$ is the energy spectrum of electrons in the QW, in the form [6]:

$\varepsilon_{n}\left(\begin{array}{l}\mathrm{r} \\ k_{\perp}\end{array}\right)=\frac{\mathrm{h}^{2} \pi^{2}}{2 m_{e} L^{2}} n^{2}+\frac{\mathrm{h}^{2} k_{\perp}^{2}}{2 m_{e}}=\varepsilon_{0} n^{2}+\frac{\mathrm{h}^{2} k_{\perp}^{2}}{2 m_{e}}=\varepsilon_{n}+\frac{\mathrm{h}^{2} k_{\perp}^{2}}{2 m_{e}}$,

where $n=0,1,2 \ldots$ and $\varepsilon_{0}=\frac{\mathrm{h}^{2} \pi^{2}}{2 m_{e} L^{2}}, L$ is the well width, $m_{e}$ is the effective mass of the electron; $\stackrel{A}{A}(t)$ is the vector potential associated with the electric field intensity vector of the laser field:

$$
\stackrel{\mathrm{r}}{A}=\stackrel{\mathrm{r}}{A_{0}} \cos (\Omega t), A_{0}=\frac{c E_{0}}{\Omega} .
$$

The Hamiltonian interaction between electron-phonon $\hat{H}_{e-a c}, \hat{H}_{e-o p}$ given by:

$$
\begin{aligned}
& \hat{H}_{e-a c}=\sum_{q} \sum_{n, n^{\prime}} G_{n, n^{\prime}}(\stackrel{\mathrm{r}}{q}) a_{n^{\prime}}^{+} a_{n}\left(b_{-q}^{+}+b_{q}^{\mathrm{r}}\right) \\
& \hat{H}_{e-o p}=\sum_{q} \sum_{n, n^{\prime}} D_{n, n^{\prime}}(\stackrel{\mathrm{r}}{q}) a_{n^{\prime}}^{+} a_{n}\left(c_{-q}^{+}+c_{q}^{\mathrm{r}}\right) .
\end{aligned}
$$


The electron-phonon interaction coefficients $G_{n^{\prime} n}(\stackrel{\mathrm{r}}{q})$, $D_{n^{\prime} n}(\stackrel{\mathrm{r}}{q})$ have form [7]:

$$
G_{n, n^{\prime}}(\stackrel{\mathrm{r}}{q})=G_{q}^{\mathrm{r}} M_{n n^{\prime}}\left(q_{z}\right), D_{n, n^{\prime}}(\stackrel{\mathrm{r}}{q})=D_{q}^{\mathrm{r}} M_{n n^{\prime}}\left(q_{z}\right)
$$

with $\quad\left|G_{q}\right|^{2}=\frac{\mathrm{h} q \xi^{2}}{2 \rho v_{a} V},\left|D_{q}^{\mathrm{r}}\right|^{2}=\frac{\mathrm{h} e^{2} v_{q}^{\mathrm{r}}}{2 V q^{2}}\left(\frac{1}{\chi_{\infty}}-\frac{1}{\chi_{0}}\right)$, $M_{n n^{\prime}}=\frac{2}{L} \int_{0}^{L} \sin \left(\frac{n \pi z}{L}\right) \sin \left(\frac{n^{\prime} \pi z}{L}\right) \exp \left(i q_{z} z\right) d z$, where $V, \rho, v_{a}, \xi$,

$\chi_{0}, \chi_{\infty}$ are the volume, the density, the acoustic velocity and the deformation potential constant, the static and high-frequency dielectric constants, respectively.

To establish a set of quantum transport equations for acoustic and optical phonons [6] we look for quantum dynamic equations for $\left\langle b_{q}\right\rangle_{t}$ :

$$
\begin{aligned}
& \frac{\partial}{\partial t}\left\langle b_{r}\right\rangle_{t}=\frac{i}{\mathrm{~h}^{2}} \sum_{n n^{\prime} k_{\perp}, s^{\prime}=-\infty} \sum_{s}^{+\infty} J_{s}(\lambda) J_{s^{\prime}}(\lambda) \exp \left(i\left(s-s^{\prime}\right) \Omega t\right)\left(f_{n}\left(\begin{array}{l}
\mathrm{r} \\
k_{\perp}
\end{array}\right)-f_{n}^{\prime}\left(\begin{array}{r}
\mathrm{r} \\
k_{\perp}-q
\end{array}\right)\right) \times \\
& \left.\times \int_{-\infty}^{t}\left\{\left|G_{n, n^{\prime}}(\stackrel{\mathrm{r}}{q})\right|^{2}\left(\left\langle b_{q}\right\rangle_{t^{\prime}}+\left\langle b_{-q}^{+\mathrm{r}}\right\rangle_{t^{\prime}}\right)+G_{n, n^{\prime}}(\stackrel{\mathrm{r}}{q)}) D_{n, n^{\prime}}(\stackrel{\mathrm{r}}{q})\left(\left\langle c_{q}^{\mathrm{r}}\right\rangle_{t^{\prime}}+\left\langle c_{-q}^{+}\right\rangle_{t^{\prime}}\right\rangle^{\prime}\right)\right\} \times \\
& \times \exp \left(\frac{i}{\mathrm{~h}}\left[\varepsilon_{n}\left(\stackrel{\mathrm{r}}{k_{\perp}}-\stackrel{\mathrm{r}}{q}\right)-\varepsilon_{n^{\prime}}\left(\begin{array}{l}
\mathrm{r} \\
k_{\perp}
\end{array}\right)+\operatorname{sh} \Omega\right]\left(t^{\prime}-t\right) d t^{\prime}\right),
\end{aligned}
$$

here $J_{s}(\lambda)$ is the Bessel function, $f_{n}\left(\begin{array}{l}\mathrm{r} \\ k_{\perp}\end{array}\right)$ is the distribution function of the electron in $|n\rangle$ state. From Eq. (8) we can obtain an equation for the Fourier transformation $B_{q}^{\mathrm{r}}(\omega)$ and $\left\langle b_{q}^{\mathrm{r}}\right\rangle_{t}$ :

$$
\begin{array}{r}
\left(\omega-\omega_{q}^{\mathrm{r}}\right) B_{q}^{\mathrm{r}}(\omega)=\frac{2}{\mathrm{~h}^{2}} \sum_{n, n^{\prime}} \sum_{=-\infty}^{+\infty}\left|G_{n, n^{\prime}}(\underset{q}{\mathrm{r}})\right|^{2} \frac{\omega_{q}^{\mathrm{r}}}{\omega-1 \Omega+\omega_{q}^{\mathrm{r}}} B_{q}(\omega-1 \Omega) P_{1}(\stackrel{\mathrm{r}}{q}, \omega)+ \\
+\frac{2}{\mathrm{~h}^{2}} \sum_{n, n^{\prime}} \sum_{1=-\infty}^{+\infty} G_{n, n^{\prime}}(-\stackrel{\mathrm{r}}{q}) D_{n, n^{\prime}}(\stackrel{\mathrm{r}}{q}) \frac{\nu_{q}^{\mathrm{r}}}{\omega-1 \Omega+v_{q}^{\mathrm{r}}} C_{\mathrm{r}}(\omega-1 \Omega) P_{1}(\stackrel{\mathrm{r}}{q}, \omega)
\end{array}
$$

Similarly for $C_{q}^{\mathrm{r}}(\omega)$ we also have:

$$
\begin{aligned}
& \left(\omega-\omega_{q}^{\mathrm{r}}\right) C_{q}(\omega)=\frac{2}{\mathrm{~h}^{2}} \sum_{n, n^{\prime}} \sum_{1=-\infty}^{+\infty}\left|D_{n, n^{\prime}}(\underset{q}{\mathrm{r}})\right|^{2} \frac{\nu_{q}^{\mathrm{r}}}{\omega-1 \Omega+v_{\mathrm{r}}} C_{q}(\omega-1 \Omega) P_{1}(\stackrel{\mathrm{r}}{q}, \omega)+ \\
& \left.+\frac{2}{\mathrm{~h}^{2}} \sum_{n, n^{\prime}} \sum_{=-\infty}^{+\infty} G_{n, n^{\prime}} \stackrel{\mathrm{r}}{q}\right) D_{n, n^{\prime}}(\stackrel{\mathrm{r}}{q}) \frac{\omega_{q}^{\mathrm{r}}}{\omega-1 \Omega+\omega_{q}^{\mathrm{r}}} B_{q}^{\mathrm{r}}(\omega-1 \Omega) P_{1}(\stackrel{\mathrm{r}}{q}, \omega)
\end{aligned}
$$

where $P_{1}(\omega, \stackrel{\mathrm{r}}{q})=\sum_{s} J_{s}(\lambda) J_{s+1}(\lambda) \Gamma_{q}^{\mathrm{r}}(\omega+s \Omega)$;

Rewriting Eqs. $(9) \div(10)$, we obtain a general dispersion equation for parametric resonance:

$$
\begin{aligned}
& {\left[\omega^{2}-\omega_{q}^{2}-\frac{2}{\mathrm{~h}^{2}} \sum_{n, n^{\prime}}\left|G_{n, n^{\prime}}(\stackrel{\mathrm{r}}{q})\right|^{2} \omega_{q}^{\mathrm{r}} P_{0}(\stackrel{\mathrm{r}}{q}, \omega)\right]\left[\omega^{2}-v_{q}^{2}-\frac{2}{\mathrm{~h}^{2}} \sum_{n, n^{\prime}}\left|D_{n, n^{\prime}}(\underset{q}{\mathrm{r}})\right|^{2} v_{q}^{\mathrm{r}} P_{0}(\underset{q}{\mathrm{r}}, \omega)\right]}
\end{aligned}
$$

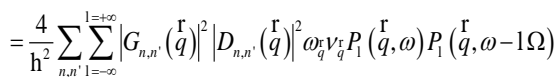

The dispersion equation, Eq. (11), is general and can be used for degenerate and non-degenerate electronic gases, for receivers of one or more photons.

To pretend that the dispersion equation, Eq. (11) is very complex, we consider, here, only the first parameter resonance case $\omega_{q}^{\mathrm{r}} \pm v_{q}^{\mathrm{r}}=\Omega$ and $\left|G_{n, n^{\prime}}(\stackrel{\mathrm{r}}{q})\right|^{2}\left|D_{n, n^{\prime}}(\stackrel{\mathrm{r}}{q})\right|^{2}<<1$, the spectrum of an acoustic phonon and an optical phonon is written in the form $\omega_{a c}(\stackrel{\mathrm{r}}{q})=\omega_{a}+i \gamma_{a} ; \omega_{o p}(\stackrel{\mathrm{r}}{q})=\omega_{o}+i \gamma_{o}$.

For an acoustic phonon, we have:

$$
\left\{\begin{array}{l}
\omega_{a} \approx \omega_{q}^{\mathrm{r}}+\frac{1}{\mathrm{~h}^{2}} \sum_{n, n^{\prime}}\left|G_{n, n^{\prime}}(\stackrel{\mathrm{r}}{q})\right|^{2} \operatorname{Re} P_{0}\left(\stackrel{\mathrm{r}}{q}, \omega_{q}^{\mathrm{r}}\right) \\
\gamma_{a}=-\frac{1}{\mathrm{~h}^{2}} \sum_{n, n^{\prime}}\left|G_{n, n^{\prime}}(\stackrel{\mathrm{r}}{q})\right|^{2} \operatorname{Im} P_{0}\left(\stackrel{\mathrm{r}}{q}, \omega_{q}^{\mathrm{r}}\right)
\end{array}\right.
$$

For an optical phonon, we have:

$$
\left\{\begin{array}{l}
v_{o} \approx \omega_{q}^{\mathrm{r}}+\frac{1}{\mathrm{~h}^{2}} \sum_{n, n^{\prime}}\left|D_{n, n^{\prime}}(\underset{\mathrm{r}}{q})\right|^{2} \operatorname{Re} P_{0}\left(\underset{q}{\mathrm{r}}, v_{q}^{\mathrm{r}}\right) \\
\gamma_{a}=-\frac{1}{\mathrm{~h}^{2}} \sum_{n, n^{\prime}}\left|D_{n, n^{\prime}}(\underset{\mathrm{r}}{q})\right|^{2} \operatorname{Im} P_{0}\left(\stackrel{\mathrm{r}}{q}, v_{q}^{\mathrm{r}}\right)
\end{array}\right.
$$

We examine dependence on $\omega$ and $q$ near the intersection $\left(\omega_{0}, q_{0}\right)$ according to the electronic interaction constant, and the phonon is [7]:

$$
\omega_{ \pm}^{ \pm}=\omega_{a}+\frac{1}{2}\left[\left(v_{a} \pm v_{0}\right) \Delta(q)-i\left(\gamma_{a}+\gamma_{0}\right) \pm \sqrt{\left[\left(v_{a} \mathrm{~m} v_{0}\right) \Delta(q)-i\left(\gamma_{a}-\gamma_{0}\right)\right]^{2} \pm \Lambda^{2}}\right]
$$

where $v_{a}\left(v_{0}\right)$ is the group velocity of acoustic phonon (optical phonon); $\omega_{a}$ is the acoustic phonon frequency renormalized due to electron-phonon interaction; $\Delta(q)=q-q_{0}$ is the intersection distance of the dispersion line; $\Delta(q)<<q$ and $\Lambda=\frac{2}{\mathrm{~h}^{2}} \sum_{n, n^{\prime}}\left|G_{n, n^{\prime}}(\stackrel{\mathrm{r}}{q})\right|\left|D_{n, n^{\prime}}(\stackrel{\mathrm{r}}{q})\right| P_{N}\left(\stackrel{\mathrm{r}}{q}, \omega_{q}^{\mathrm{r}}\right)$.

The condition for an increase in acoustic phonon parameters is that the imaginary parts of $\omega_{ \pm}^{ \pm}$must be positive, ie:

$$
\operatorname{Im} \omega_{+}^{-}=\frac{1}{2}\left(-\left(\gamma_{a}+\gamma_{0}\right)+\sqrt{\left(\gamma_{a}-\gamma_{0}\right)^{2}+\Lambda^{2}}\right)>0
$$

When thermal energy $k_{B} T$ is much smaller than Fermi energy, the electronic gas degrades. The condition of the electromagnetic field amplitude is an increase in the acoustic phonon:

$$
\begin{aligned}
& E_{t h}=\frac{2 m^{* 3} \Omega}{e h^{4} q^{3}} \frac{\varepsilon_{m n^{\prime}}\left(\omega_{q}^{r}\right)\left(\varepsilon_{m r^{\prime}}\left(\omega_{q}^{\prime}\right)+\mathrm{h} \Omega\right)}{\sqrt{2 m^{*}\left(\varepsilon_{F}-\varepsilon_{n^{\prime}}\right)}-\sqrt{2 m^{*}\left(\varepsilon_{F}-\varepsilon_{n}-\sigma q^{2}\right)}} \times \\
& \times\left\{\left[\frac{2 \mathrm{~h}^{2} q^{2}}{m^{*}}\left(\varepsilon_{F}-\varepsilon_{n^{\prime}}\right)-\varepsilon_{n n^{\prime}}^{2}\left(\nu_{q}^{\mathrm{r}}\right)\right]^{\frac{1}{2}}-\left[\frac{2 \mathrm{~h}^{2} q^{2}}{m^{*}}\left(\varepsilon_{F}-\varepsilon_{n^{\prime}}+\mathrm{h} \nu_{q}^{\mathrm{r}}\right)-\varepsilon_{m n^{\prime}}^{2}\left(v_{q}^{\mathrm{r}}\right)\right]^{\frac{1}{2}}\right\}^{\frac{1}{2}} \\
& \times\left\{\left[\frac{2 \mathrm{~h}^{2} q^{2}}{m^{*}}\left(\varepsilon_{F}-\varepsilon_{n^{\prime}}\right)-\varepsilon_{m n^{\prime}}^{2}\left(\omega_{q}^{\mathrm{r}}\right)\right]^{\frac{1}{2}}-\left[\frac{2 \mathrm{~h}^{2} q^{2}}{m^{*}}\left(\varepsilon_{F}-\varepsilon_{n^{\prime}}+\mathrm{h} \omega_{q}^{\mathrm{r}}\right)-\varepsilon_{m n^{\prime}}^{2}\left(\omega_{q}^{\mathrm{r}}\right)\right]^{\frac{1}{2}}\right\}^{\frac{1}{2}}
\end{aligned}
$$

where $\varepsilon_{n n^{\prime}}(\omega)=\varepsilon_{n^{\prime}}-\varepsilon_{n}-\mathrm{h} \omega-\sigma q^{2} ; \sigma=\frac{\mathrm{h}^{2}}{2 m_{e}}$.

In order to clarify the mechanism for parametric acoustic-phonon amplification, we calculate the threshold amplitude for the parametric amplification of acoustic phonons in a GaAs/GaAlAs QW. The parameters used in the calculation are as follows [8]: $\xi=13.5 \mathrm{eV}, \rho=5.32$ $\mathrm{gcm}^{-3}, \mathrm{v}_{\mathrm{a}}=5370 \mathrm{~ms}^{-1}, \chi_{0}=12.9, \chi_{\infty}=10.9, \mathrm{~d}=100 \mathrm{~A}^{0}$, 
$\varepsilon_{\mathrm{F}}=0.05 \mathrm{eV}, \mathrm{m}_{\mathrm{e}}=0.066 \mathrm{~m}_{0}, \mathrm{~m}_{0}$ being the mass of a free electron, and the optical phonon energy $\mathrm{h} v=36.25 \mathrm{meV}$.

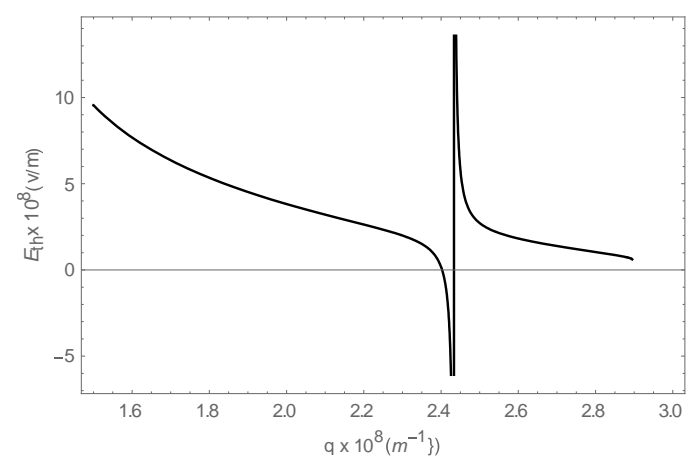

Fig. 1. Dependence on the wave number $\mathrm{q}$ of the threshold amplitude of the $\mathrm{E}_{\mathrm{th}}$ field.

In Figure 1, we see around $\mathrm{q}=2.45 .10^{8} \mathrm{~m}^{-1}$ with the region at which the threshold amplitude value is negative. This is due to the fact that in the parametric resonance condition there is an increase in acoustic phonons, accompanied by the process of annihilating acoustic phonons. It means the transmission of acoustic phonons energy to optical phonons and vice versa has the effect of an external laser field. The process of canceling an acoustic phonon should occur, so there is no need to supply the energy of an external field.

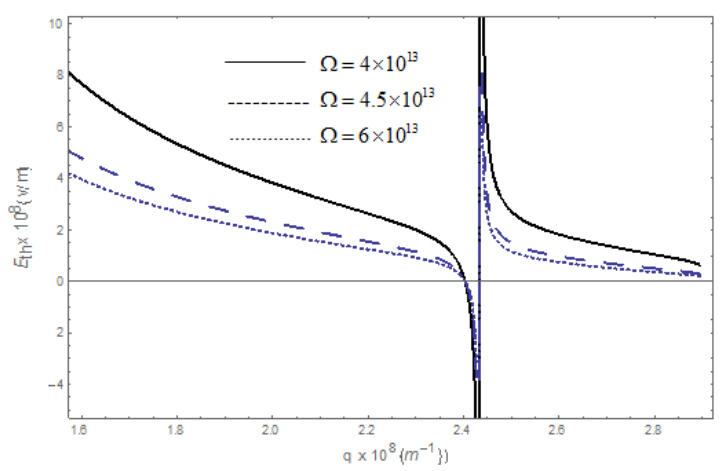

Fig. 2. Dependence on the wave number q of the threshold amplitude of the $\mathrm{E}_{\mathrm{th}}$ field with different values of external field frequency.

Figure 2 shows that the threshold amplitude of the field is higher when we increase external field frequency.

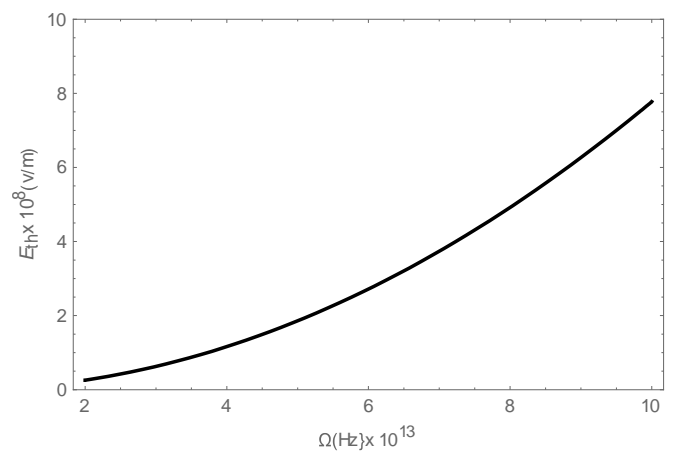

Fig. 3. Dependence on the external field frequency of threshold amplitude of the $\mathrm{E}_{\mathrm{th}}$ field.
In Figure 3 with wave $\mathrm{q}=2 \times 10^{8} \mathrm{~m}^{-1}$, we see that as the frequency of the laser field increases, the threshold amplitude also increases. We continue to examine the threshold field number in terms of number waves.

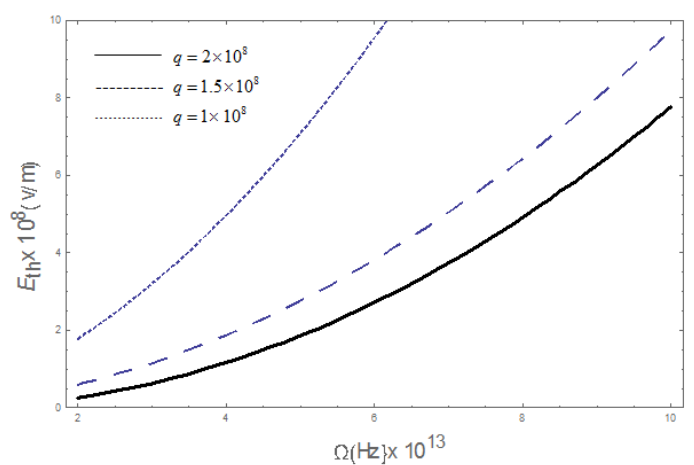

Fig 4. Dependence on the external field frequency of the threshold amplitude of the $E_{\text {th }}$ field with different values of the waves number.

According to Figure 4, we see that with the same laser field frequency value, the threshold amplitude value increases sharply with smaller wave q numbers. For a laser source with a defined frequency, in order to have parameter resonance, we must select a laser source whose amplitude is greater than the threshold amplitude value.

In this paper, we analytically investigated the possibility of parametric resonance of acoustic and optical phonons in a QW. We obtained a general dispersion equation for parametric amplification and transformation of phonons and the threshold amplitude of the field for acoustic phonon parametric amplification. The parametric amplification for acoustic phonons in a QW could occur under the condition that the amplitude of an external electromagnetic field is higher than the threshold amplitude.

\section{References}

[1] B.A. Glavin, V.A. Kochelap, T.L. Linnik, P. Walker, A.J. Kent, M. Henini, J. Physics: Conference Series 92, (2007).

[2] O.A.C. Nunes, J. Appl. Phys. 115, 233715 (2014).

[3] Yu. E. Lozovik, S.P. Merkulova, I.V. Ovchinnikov, Phys. Lett. A 282, 407 (2001).

[4] R.P. Beardsley, A.V. Akimov, M. Henini, A.J. Kent, Phys. Rev. Lett. 104, 085501 (2010).

[5] P. Ruello, V.E. Gusev, Ultrasonics 56, 21 (2015).

[6] L. Esaki, in Proc. 17th Int. Conf. Phys. Semiconductors, San Francisco, CA, Aug, J.D. Chadi and W.A. Harrison, Eds, Berlin: Springer- Verlag 473 (1984).

[7] W. Xu, F.M. Peeters, J. T. Devreese, Phys. Rev. B 48, 1562 (1993).

[8] T.C. Phong, N.Q. Bau, J. Korean Phys. Soc. 42(5), 647 (2003). 